Ciencia y Salud, Vol. II, No. 3, septiembre-diciembre, 2018 • ISSN: 2613-8816 | E-ISSN: 2613-8824

DOI: https://doi.org/10.22206/cysa.2018.v2i3.pp63-68

\title{
NESIDIOBLASTOSIS EN UNA ESCOLAR, A PROPÓSITO DE UN CASO
}

\author{
Nesidioblastosis in a school, about a case
}

\section{Rosario Almánzar ${ }^{1}$, Adonise Rosario² ${ }^{2}$ Zaymi Díaz $^{3}$, Jiomar Figueroa ${ }^{4}$, Massiel Segura $^{5}$}

\author{
Recibido: 6 marzo 2018 Aprobado: 23 mayo 2018
}

\begin{abstract}
Cómo citar: Almanzar R, Rosario A, Diaz Z, Figueroa J, Segura M. Nesidioblastosis en una escolar, a propósito de un caso. Ciencia Y Salud [Internet]. 14sep.2018 [citado 14 sep.2018]; 2(3):63-8. Available from: https://revistas.intec.edu.do/index.php/cisa/ article/view/1287
\end{abstract}

\section{Resumen:}

Introducción: La nesdioblastosis se define como proliferación de las células endocrinas pancreáticas, que comporta alteraciones en su distribución y diferenciación. El número de los islotes de Langerhans es menor de lo normal, encontrándose las células endocrinas distribuidas, anárquicamente, por el parénquima pancreático, en forma de pequeños nidos o cordones ${ }^{1}$.

1- Pediatra endocrinóloga, gerente de Servicio de Endocrinología, Hospital Infantil Robert Reid Cabral, profesora del INTEC.

2- Pediatra endocrinóloga, Hospital Infantil Robert Reid Cabral, Servicio de Endocrinología.

3- Pediatra Cirujana, Hospital Infantil Robert Reid Cabral, Servicio de Cirugía.

4- Cirujano transplantólogo, Hospital General Plaza de la Salud, transplantólogo.

5- Residente de 3 año de Endocrinología Pediátrica, Hospital Infantil Robert Reid Cabral, Servicio de Endocrinología.

Emails:

rosario.almanzar@intec.edu.do

adonise.rosario@gmail.com

zadadiva@gmail.com

jfigueroa@yahoo.com

dramaxsegura@gmail.com
Objetivo: Describir un caso clínico de nesidioblastosis en una paciente en edad escolar, con historia de convulsiones tonicoclónicas desde 1 año de edad, tratado con Oxcarbazepina. Presentando hipoglucemias sintomáticas desde 1 año y medio, por lo que fue referida a la consulta de endocrinología.

Presentación del caso clínico: Paciente femenina de 7 ańos y 5 meses de edad cronológica, referida al departamento de endocrinología con historia de enfermedad actual, caracterizada diaforesis y mareos; presenta movimientos tónico-clónicos generalizados, retroversión ocular con pérdida del estado de conciencia (en 2 oportunidades), es trasladada a centro médico donde evidencian glucemia en 18-11-32-16 mg/dl respectivamente, colocan solución dextrosa con mejoría momentánea. Permanece por 9 días sin recuperación completa y aparecen fiebre de 3 días de evolución; Es trasladada al Hospital Infantil Robert Reid Cabral (RRC), donde se estudia y se confirman las hipoglucemias, analíticas que revelan hormonas contrarreguladoras elevadas, niveles de insulina en 2,700 mui/ml. Se estabiliza con octreotide y se decide llevar a cirugía para pancreactectomia, realizándose laparotomía abierta. Biopsia trans-operatoria reportó: hiperplasia de las células de los islotes de Langerhans. Por lo que se extirpa un $95 \%$ de tejido pancreático, presenta hiperglicemias y se inicia tratamiento con análogos de insulina de acción 
lenta $(0.14 \mathrm{ud} / \mathrm{kg} / \mathrm{d})$, enzimas pancreáticas 10,000ud, 15 min previo a comidas y multivitamínicos.

Al mes de la cirugía la paciente vuelve a presentar signos de hipoglicemias, se suspende la insulina y se ingresa, se verifican las hipoglicemias y se realiza una segunda intervención quirúrgica. También se realiza excéresis de 5\% de páncrea; segundada porción del duodeno, con anastomosis terminal. Actualmente responde al tx con insulina análoga.

Conclusión: La nesidioblastosis en una causa rara de hipoglucemia por hiperinsulinismo endógeno en el escolar, clínicamente es indistinguible del insulinoma; bioquímicamente es necesario documentar la hipoglucemia hiperinsulinémica durante el ayuno, el tratamiento de elección es la cirugía y seguimiento médico a los déficits secundarios a la misma.

Palabras claves: Nesidioblastosis, hipoglucemia, hiperinsulinismo, pancreatectomía. Hiperglicemia.

\section{Abstract}

Introduction: Necidioblastosis is defined as proliferation of pancreatic endocrine cells, which involves alterations in their distribution and differentiation. The number of the islets of Langerhans is lower than normal, with the endocrine cells distributed anarchically by the pancreatic parenchyma in the form of small nests or cords ${ }^{1}$.

Objective: To describe a clinical case of nesidioblastosis in a patient of school age, with a history of tonic-clonic seizures from 1 year of age, treated with Oxcarbazepine. Presenting symptomatic hypoglycaemia from 1 year and a half, so it was referred to the endocrinology clinic.

Presentation of the clinical case: Female patient of 7 years and 5 months of chronological age, referred to the department of endocrinology with a history of current illness, characterized by diaphoresis and dizziness; presents generalized tonic-clonic movements, ocular retroversion with loss of consciousness (on 2 occasions), is transferred to a medical center where they demonstrate glycemia at 18-11-32-16 mg / dl respectively, place dextrose solution with momentary improvement. It remains for 9 days without complete recovery and fever appears 3 days of evolution and is transferred to the Robert Reid Cabral Children's Hospital, where it is studied and confirmed hypoglycemia, laboratory tests that reveal high counter-regulating hormones, insulin levels in 2,700 mui
/ ml, stabilizes With octreotide and it is decided to carry out surgery for pancreactectomy, open laparotomy is performed. Trans-operative biopsy reported: hyperplasia of the islet cells of Langerhans. So $95 \%$ of pancreatic tissue is removed, presents hyperglycemia and treatment is started with slim-action insulin analogs $(0.14 \mathrm{ud} / \mathrm{kg} /$ d) pancreatic enzymes $10,000 \mathrm{~d}, 15 \mathrm{~min}$ prior to meals, multivitamin. One month after surgery, the patient presented signs of hypoglycemia again, insulin was discontinued and the patient was admitted, hypoglycemia was verified, and second surgical intervention was performed. An excess of $5 \%$ of the pancreas and 2 nd portion of the duodenum was performed with an end-to-end anastomosis. Currently responding to tx with analogous insulin.

Conclusion: Nesidioblastosis in a rare cause of hypoglycaemia due to endogenous hyperinsulinism in the school, clinically is indistinguishable from insulinoma; biochemically it is necessary to document hyperinsulinemic hypoglycaemia during fasting, the treatment of choice is surgery and medical treatment to the deficits 2 nd to it.

Key words: Nesidioblastosis, hypoglycemia, hyperinsulinism, pancreatectomy. Hyperglycemia

\section{Introducción}

La necidioblastosis se define como proliferación de las células endocrinas pancreáticas, que comporta alteraciones en su distribución y diferenciación. El número de los islotes de Langerhans es menor de lo normal, encontrándose las células endocrinas distribuidas, anárquicamente, por el parénquima pancreático, en forma de pequeños nidos o cordones ${ }^{1}$.

La hipoglucemia hiperinsulinémica endógena (HHE) puede ser causada por tumores sólidos del páncreas que secretan excesiva cantidad de insulina, conocidos como insulinomas, menos comúnmente por una hiperplasia de las células de los islotes de Langerhans, conocida como nesidioblatosis ${ }^{1}$ y rara vez por tumores secretores de factor de crecimiento insulinoide tipo 2 ( $\mathrm{IGF}-2$ ), tales como mielomas, linfomas y leucemias. La nesidioblastosis fue inicialmente reportada por George F. Laidlaw en 
1938 2. La incidencia en niños se estima en 0.5 casos/año, sin diferencia entre sexos ${ }^{3}$.

Actualmente, gracias al trabajo de muchos investigadores, se han notificado 8 partes en el organismo, asociados al hiperinsulinismo: ABCC8, KCNJ11, HADH1, GCK, GLUD1, SLC16A1, UCP2 y HNF4a. Las mutaciones de estos sitios tienen diferencias importantes en el fenotipo y el modelo de herencia 5 .

$\mathrm{Al}$ respecto, los dos primeros, $\mathrm{ABCC} 8$ y genes de KCNJ11, se localizan juntos en el brazo corto del cromosoma 11, e involucra a los que presentan alteraciones del canal de potasio en la célula beta, lo cual la conduce a su despolarización con el ingreso de calcio y la consiguiente liberación de insulina. Por otra parte, el canal de potasio más adenosintrifosfato está formado por 2 pares de proteínas denominadas sulfonilureas (Sur-1 y Kir-6.2). Estas 4 subunidades forman un canal de potasio que se cierra en presencia de Atp. Dicha relación de defectos del receptor de sulfonilureas en la membrana de las células beta, con la consecuente alteración del flujo de potasio (ya mencionado) y el incremento de insulina no regulada por los mecanismos habituales de retroalimentación, da como resultado la hipoglucemia persistente ${ }^{5}$.

\section{Caso clínico}

Femenina de 7 años y 5 meses de edad, con historia de convulsiones tónico clónicas desde 1 año de edad, tratado con Oxcarbazepina; presentahipoglucemias sintomáticas al ańo y medio, por lo que su médico inició tratamiento con L- CARNITINA desde entonces, y hasta la fecha. A los 4 años se le suspenden anticonvulsivantes. Es referida desde otro centro de salud en Puerto Plata y tratada por endocrinólogo de adultos, luego de un internamiento por 9 días por hipoglucemias severas (18$11-32-16 \mathrm{mg} / \mathrm{dl}$...) y fiebre de 3 días de evolución, en condiciones generales muy grave.
Examen físico: sudoración profusa, sensación de mareos, temblor, estupor.

Tiroides: grado 0; Axilar: III Olor apócrino, Mamas: tanner I; Púbico: tanner II. Piel: suave. Patrón evacuatorio: diario.

Peso: $33.7 \mathrm{~kg}$ (percentil 90-95). Talla: $123 \mathrm{~cm}$ (percentil 50). IMC: $22 \mathrm{~kg} / \mathrm{m} 2$ (percentil $>97$ correspondiendo a obesidad I) es trasladada al hospital infantil RRC, donde se estudia y se confirman las hipoglucemias, analíticas que revelan hormonas contrarreguladoras elevadas, niveles de insulina en $2,700 \mathrm{mui} / \mathrm{ml}$ en presencia de hipoglucemia 45 $\mathrm{mg} / \mathrm{dl}$.

\section{Cuadro de analíticas realizadas}

\begin{tabular}{ll}
\hline HEMOGRAMA: & LEU 8.05 MM3/HB 11.3/ \\
& HCTO 34.1\%/363 MM3 \\
GLUCEMIA BASAL: & $45 \mathrm{mg} / \mathrm{dl}$ ( 65-100) \\
GLUCEMIA POST GLU- & $64 \mathrm{mg} / \mathrm{dl}$ \\
COLA: & \\
INSULINA BASAL: & $90 \mathrm{uui} / 355 \mathrm{ug} / \mathrm{dl}$ ( 5-25)ml (0- \\
& $29)$ \\
CORTISOL BASAL: & $355 \mathrm{ug} / \mathrm{dl}(5-25)$ \\
PÉPTIDO C BASAL: & $0.17 \mathrm{ng} / \mathrm{ml}(0.90-7.10)$ \\
HbA1c: & $5 \%(4.3-5.8)$ \\
HORMONA DE CREC- & $7.59 \mathrm{ng} / \mathrm{ml}(0-8)$ \\
IMIENTO: & \\
TSH ULTRA SENSIBLE: & $4.36 \mathrm{uui} / \mathrm{ml}$ \\
T4LIBRE & $1.24 \mathrm{ng} / \mathrm{dl}$ \\
AMONIO: & $1.21 \mathrm{ug} / \mathrm{ml}(0.20-1.21)$ \\
LDH: & $214 \mathrm{u} / \mathrm{l}(141-237)$ \\
DHEA-S: & $33.6 \mathrm{ug} / \mathrm{dl}(35-430)$ \\
ORINA: & Negativa \\
COPROLÓGICO: & Negativo \\
\hline
\end{tabular}

Se realizan estudios por imágenes, como: sonografía abdominal, tomografía axial computarizada abdominal y resonancia magnética abdominal sin hallazgos patológicos.

Se corrige hipoglucemias con flujo de dextrosa $7 \mathrm{mg} / \mathrm{kg} / \mathrm{min}$ y octreótide vía parenteral, luego de estabilizar a paciente y dar de alta para continuar 
investigación del caso, se indica iazóxide jarabe para manejo ambulatorio. Sin embargo, este no lo administran, paciente vuelve a presentar hipoglucemias severas, acompañadas en dos ocasiones por eventos convulsivos. Se realizan analíticas y el resultado de Insulina basal es de 2,700 ui/ml, por lo que se decide optar por el tratamiento definitivo de las hipoglucemias secundarias a hiperinsulinismo: pancreatectomía para realizar biopsia por congelación. Se autoriza el procedimiento quirúrgico, y se realizan las evaluaciones prequirúrgicas correspondientes.

Informe quirúrgico: durante procedimiento se constata páncreas de gran tamaño por lo que se decide realizar Pancreatectomía Subtotal en un 95\%.

\section{Reporte de biopsia tranquirúrgica:}

De tejido de cabeza de páncreas: tejido pancreático benigno con distorsión de los islotes de langerhans y presencia de células morfológicamente de tipo beta adyacente a los acinos serosos en el interior de los islotes bien delimitados. Estos hallazgos sugieren Nesidioblastosis. Sin embargo, se recomienda confirmar la presencia de células beta fuera de los islotes, por medio de inmunohistoquímica, ganglio pre arteria esplénica: ganglio linfático con hiperplasia linfoide reactiva benigna.

Paciente evoluciona satisfactoriamente y se egresa en condiciones generales estable, con tratamiento de:

- Análogos de insulina de acción lenta (0.14 $\mathrm{ud} / \mathrm{kg} / \mathrm{d}$ ).

- Enzimas pancreáticas 10,000ud, 15 min previo a comidas.

- Multivitamínicos.

En las próximas dos semanas la paciente vuelve a presentar manifestaciones clínicas de hipoglicemia, mareos, temblor, pérdida de conocimiento y sudoración profusa, se suspende la insulina y al comprobarse las hipoglicemias se decide llevar nuevamente a quirófano y retirar el 5\% restante de tejido pancreático. Actualmente en condiciones generales estables, la paciente no ha vuelto a presentar sintomatologías de hipoglucemia, reiniciando la insulina y continuando con enzimas pancreáticas y multivitamínicas.

\section{Discusión}

La hipoglicemia severa (por hiperinsulinismo endógeno) que amenaza la vida y que mejora momentáneamente con el aporte de glucosa, son características clínicas típicas del Insulinoma y la nesidioblastosis. Es difícil establecer la diferencia entre estas entidades desde el punto de vista clínico. Una vez que se ha excluido la enfermedad facticia del uso inapropiado de insulina o sulfonilurea ${ }^{7}$, el diagnóstico es eminentemente histológico. Para nesidioblastosis los criterios son:

a. aumento del tamaño y número de las células $\bigotimes$ de los islotes de Langerhans, b. incremento del número de islotes periductales, c. núcleo hipercromático, d.-abundante citoplasma claro, e. exclusión microscópica e inmunohistoquímica de un insulinoma. ${ }^{2}$ De estas características las primeras dos coinciden con la histología de nuestra paciente, lo que confirma el diagnóstico de nesidioblastosis.

Observamos en los estudios de imagen que con frecuencia no permiten evidenciar la lesión, siendo negativos los hallazgos en la nesidioblastosis, estudios como: sonografía abdominal, tomografía axial computarizada de abdomen y ecoendoscopia; se reportan sin hallazgos ${ }^{2}$, típico en la nesidioblastosis.

En el insulinoma, las pruebas de imágenes presentan una alta sensibilidad, principalmente en lesiones mayores de $2 \mathrm{cms}$, y localizadas en la cabeza y cuerpo del páncreas (TAC abdominal con contraste y multicortes con sensibilidad del $95 \%$, ecoendoscopia sensibilidad 99\%). ${ }^{2}$ 
Documentación de nivel de glucosa en sangre $<50$ $\mathrm{mg} / \mathrm{dl}$ con síntomas de hipoglucemia; alivio de los síntomas después de comer, aumento del nivel de insulina en plasma ( $\geq 6 \mu \mathrm{U} / \mathrm{ml}$ ), aumento del nivel de péptido $C(\geq 0.2 \mathrm{nmol} / \mathrm{l})$, aumento del nivel de proinsulina ( $\geq 5 \mathrm{pmol} / \mathrm{l})$, y ausencia de sulfonilurea plasmática.

Algunos autores sugieren un componente genético en la etiología de la Nesidioblastosis, como alteración en la expresión de los genes ABCC8 (SUR 1), KCNJ11 (Kir 6.2), GCK, GLUD1; en recién nacidos $^{7}$, y en adultos, mutaciones secundarias a un patrón de herencia autosómico dominante, autosómico recesivo o a mutaciones de novo de los genes del SUR1 (gen ABCC8), o del canal recticador de potasio Kir 6.2 (gen KCNJ11) ${ }^{11}$.

En aquellos pacientes donde existe la sospecha clínica de un patrón heredo-familiar (al menos un familiar afectado en primer grado), se sugiere el estudio genético de consejería pre-concepcional para las próximas gestaciones; el estudio genético no está aún estandarizado para esta enfermedad, en el caso descrito no existen antecedentes familiares de sospecha para pensar en un componente heredo-familiar, por lo cual no fueron solicitados los estudios genéticos previamente señalados.

\section{Conclusión:}

La nesidioblastosis es una causa rara de hipoglucemia hiperinsulinémica en la edad escolar. Los síntomas clínicos se basan en la presencia de la triada de Whipple (síntomas de hipoglucemia, concentraciones de glucemia venosa disminuida, desaparición de síntomas tras la normalización de la glucemia); y son indistinguibles del insulinoma. Los estudios de imágenes no aportan datos específicos, los análisis de laboratorio se basan en la documentación del hiperinsulinismo endógeno. El diagnóstico es histológico, con cambios en la morfología de las células $\beta$ dadas por la hiperplasia e hipertrofia de las mismas, núcleos hipercrómicos y citoplasma claro abundante. El tratamiento es quirúrgico, siendo recomendada la pancreatectomía casi total por su bajo riesgo de recidiva y la terapia de sustitución con enzimas pancráticas e insulina.

\section{Referencias}

1. Clínica Universidad de Navarra. Diccionario médico [Internet] Navarra, Clínica Universidad de Navarra, [citado 2018 mar]. Disponible en http://www.cun.es/diccionario-medico/ terminos/Nesidioblastosis

2. De Jesús J, Fung L, García F, Núñez. Nesidioblastosis en adolescentes: A propósito de un caso. Rev. Venez. Endocrinol. Metab. [Internet]. 2015 Mar [citado 2018 Abr 17];13(1):4853. Disponible en: http://www.scielo. org.ve/scielo.php?script $=$ sci_arttext $\&$ pi$\mathrm{d}=$ S1690-31102015000100006\&lng=es.

3. Antunes JD, Gefner ME, Lippe BM, Lanau BM. Hypoglycaemia in children: differentiating hyperinsulinaemic from nonhyperinsulinaemic causes. J Pediatr. 1990;116:990-994

4. Then C, Apostolopoulos Y, Seissler J, Lechner A. Refractory Idiopatic Non- Insulinoma Pacreatogenous Hypoglycemia in an adult: Case Report and Review of the literature. J Pancreas. 2013;14:264-268.

5. Zaldívar Ochoa JR, Rodríguez Carballo A, Quesada CM, Martínez AM, Santiago Oconnor A, Menéndez Rodríguez $M$. Nesidioblastosis: hipoglucemia hiperinsulínica persistente en un recién nacido. MEDISAN [Internet]. $2012 \mathrm{Dic}$ [citado $2018 \mathrm{Abr}$ 16];16(12):1948-1953. Disponible en: http:// scielo.sld.cu/scielo.php?script=sci_arttext\&pi$\mathrm{d}=$ S1029-30192012001200019\&lng=es. 
6. Shin, JJ, Gorden P, Libutti SK. Insulinoma: pathophysiology, localization and management. Future Oncology. 2010;6(2):229-237. Disponible en http://doi.org/10.2217/ fon.09.165,

7- Thompson SM, Vella A, Thompson GB, et al. Selective Arterial Calcium Stimulation With
Hepatic Venous Sampling Differentiates Insulinoma From Nesidioblastosis. The Journal of Clinical Endocrinology and Metabolism. 2015;100(11):4189-4197. doi:10.1210/ jc.2015-2404 